\title{
Using the Pattern Function of Angular Momentum Improve the Directivity of Binary Antenna Array
}

\author{
Jiangmei Tang, Bin Yao and Qinhong Zheng \\ College of Physical Science and Electronic information, Yunnan Normal university., Kunming 650500, China
}

\begin{abstract}
Building solar power stations in space are concerned by international .In the process of electricity transmission to need to make the solar cell array always orient for the sun. The microwave beam of transmitting antenna must point to ground receiving antenna, which requirement the antenna has the very good directivity. In this article, under the condition of the pattern function of angular momentum deduced the directivity coefficient of binary antenna array. Using Matlab software simulation probed the situation of binary antenna array directivity improvement. The simulation results show that in some specific values the directivity of binary antenna array get enormously improvement. In some specific areas the directivity of binary antenna array get obvious improvement. The maximum value of directivity coefficient is 283.11.
\end{abstract}

Keywords-pattern function; quantization; binary antenna array; directivity

\section{INTRODUCTION}

The sunlight intensity in space five to ten times greater than the ground. Solar power generation technology can provide constant energy without pollution, Space solar power mainly includes three parts: solar launchers device, energy conversion and launchers device, ground receiving and conversion device. Energy conversion device convert energy into microwave. Using the energy launchers device will microwave launch back ground. Launch device is made using the antenna. Microwave beam of the auncher antenna must point to ground receiving antenna, which requires the antennah as the very good directivity[1]. Directivity coefficient as a parameter is used to represent the antenna to a certain direction concentration degree for the radiation of electromagnetic wave (that is the sharp degree of the directivity diagram). With the development of the antenna theory, there are a number of array antenna directivity researches. In recent years, the emergence of a variety of numerical integration method $[2,3,4,5,6]$ can synthesis irregular array, and get the specified sidelobe envelope, but these methods are not under the condition of control the sidelobe and to consider implementing optimal array directivity at the same time. Sanzgiri and Bulter [7] based on eigenvalue decomposition method, under the condition of the binary antenna array optimization array directivity and control the sidelobe pattern at the same time. But the method convergence is slow and there is no limit of sidelobe phase which has a great influence on the final result. Yong-feng Chen , Xing-zhong Huang a piece work in 2007[8], quadratic synthetic method, the constraint optimization synthesis, Dolph-Chebyshev three synthesis methods were introduced in detail and finally suggests that no matter from the point of flexibility or calculation accuracy binding optimization method can better solve the comprehensive problem of antenna sidelobe constraint conditions and efficiency conditions. Although people put forward to kinds of optimizations of antenna directivity, but the array antenna directivity coefficient not got enormously improvement. With the advent of quantum communication, antenna will also be toward "quantum" the direction developmen, using the method of quantized directional coefficient optimized antenna array have great significance.

At first, this paper under the condition of the angular momentum of the pattern function deduced the directivity coefficient of binary antenna array. Then used the Matlab software simulation to explore the improvement situation of the dual antenna directivity. Founding binary array antenna directivity get unprecedented improvement.

\section{The Direction Function of the ANGUlar MoMentum THE BINARY ARRAY ANTENNA DIRECTIVITY COEFFICIENT}

$\mathrm{N}$ yuan array antenna directivity function

$$
S(\theta, \varphi)=\sum_{n=0}^{N-1} I_{n} e^{j k Z_{n} \cos \theta}
$$

According to the [9]

$$
\begin{aligned}
& e^{j k r \cos \theta}=\sum_{l=0}^{\infty}(2 l+1) i^{l} j_{l}(k r) P_{l}(\cos \vartheta) \\
& =\sum_{l=0}^{\infty} \sqrt{4 \pi(2 l+1)} i^{l} j_{l}(k r) Y_{l 0}(\theta)
\end{aligned}
$$

The angular momentum of the eq (1) is changed into

$$
S(\theta, \varphi)=\sum_{n=0}^{N-1} I_{n} \sum_{l=0}^{m} \sqrt{4 \pi(2 l+1) i} l j_{l}\left(k Z_{n}\right) Y_{l 0}(\theta)
$$

Among them $l=0,1,2 \ldots$, Antenna directivity coefficients 


$$
D=\frac{4 \pi}{\Omega_{A}}=\frac{4 \pi S\left(\theta_{0}, \varphi_{0}\right) S^{*}\left(\theta_{0}, \varphi_{0}\right)}{\iint S(\theta, \varphi) S^{*}(\theta, \varphi) \sin \theta d \theta d \varphi}
$$

$\left(\theta_{0}, \varphi_{0}\right)$ is main lobe location

III. The BinARY ARRAY ANTENNA Directivity COEFFICIENTS

Binary antenna array model is displayed in figure I

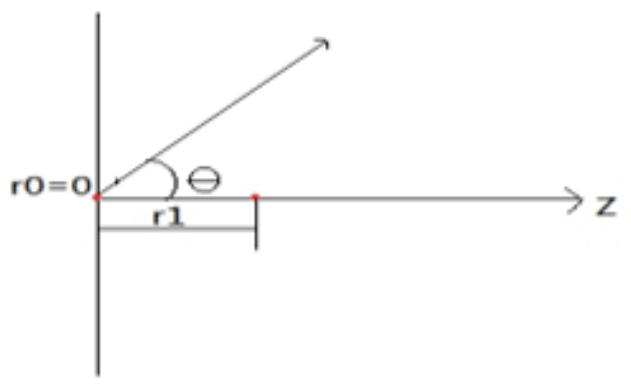

FIGURE I. BINARY ARRAY ANTENNA CALCULATION MODEL

For $^{N=2, l=1}$ binary antenna array (3) is changed into

$$
\begin{aligned}
& S(\theta, \varphi)=I_{0}\left[j_{0}\left(k r_{0}\right)+3 i j_{1}\left(k r_{0}\right) \cos \theta\right] \\
& +I_{1}\left[j_{0}\left(k r_{1}\right)+3 i j_{1}(k r 1) \cos \theta\right]
\end{aligned}
$$

Making $\theta_{0}=0$ is main lobe location. The binary array antenna directivity coefficients is changed into

$$
\begin{aligned}
& D_{1}=\frac{4 \pi}{\Omega A}=\frac{4 \pi S\left(\theta_{0}, \varphi_{0}\right) S^{*}\left(\theta_{0}, \varphi_{0}\right)}{\iint S(\theta, \varphi) S^{*}(\theta, \varphi) \sin \theta d \theta d \varphi} \\
& S\left(\theta_{0}, \varphi_{0}\right)=I_{0}\left[j_{0}\left(k r_{0}\right)+3 i j_{1}\left(k r_{0}\right)\right] \\
& +I_{1}\left[j_{0}\left(k r_{1}\right)+3 i j_{1}(k r 1)\right] \\
& \quad S^{*}\left(\theta_{0}, \varphi_{0}\right)=I_{0}\left[j_{0}\left(k r_{0}\right)-3 i j_{1}\left(k r_{0}\right)\right] \\
& +I_{1}\left[j_{0}\left(k r_{1}\right)-3 i j_{1}(k r 1)\right]
\end{aligned}
$$

Make(5), (7), (8), (9) into (6) and get $\theta_{0}=0, \varphi_{0}=0$

The directivity coefficients of pattern function without changing is ${ }^{D_{2}}$ and get $\theta_{0}=0, \varphi_{0}=0$

$$
\begin{aligned}
& D_{2}=\frac{4 \pi}{\Omega A}
\end{aligned}
$$

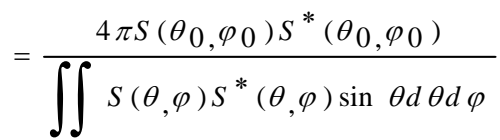

$$
\begin{aligned}
& S^{*}\left(\theta_{\left.0, \varphi_{0}\right)}\right) \sum_{n=0}^{N-1} I_{n} e^{-j k Z} \\
& S\left(\theta_{0}, \varphi_{0}\right)=\sum_{n=0}^{N-1} I_{n} e^{j k Z_{n}} \\
& S^{*}\left(\theta_{\left.0, \varphi_{0}\right)}\right) \sum_{n=0}^{N-1} I_{n} e^{-j k Z_{n} \cos \theta}
\end{aligned}
$$

Make(1), (11), (12), (13)into(10)can to get ${ }^{D_{2}}$.

\section{The Matlab Simulation Results AND DISCUSSION}

When $\theta_{0}, \varphi_{0}, r_{0}, r_{1}$ take fixed value using Matlab software to draw the simulation diagram of ${ }^{D_{1}, D_{2}}$ with the change of $\theta$ is shown in figure II. Through figure II known when ${ }^{\theta}$ get $0 \sim 28.8 .47 .80 \sim 58.78 .75 .52 \sim 81.61$,

$98.50 \sim 104.60 .121 .30 \sim 132.27 .151 .30 \sim 180$ degrees these areas $D_{1}$ is obviously improved.

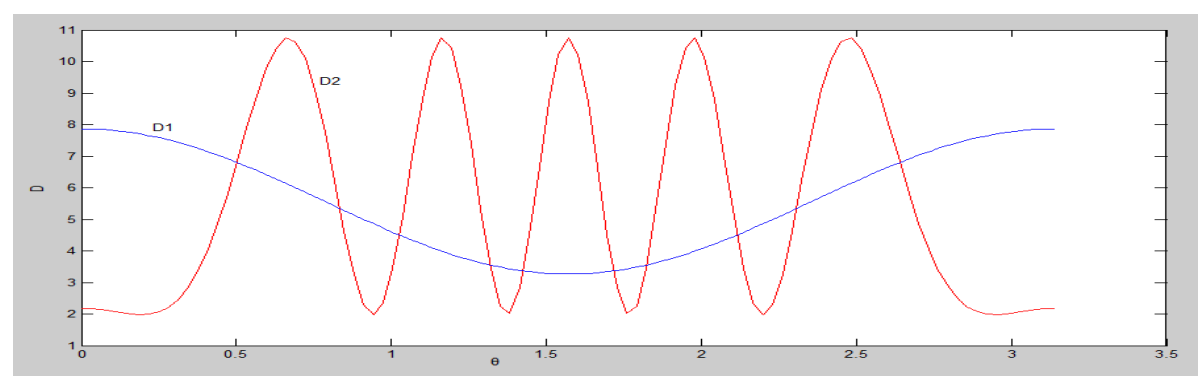

FIGURE II. WITH THE CHANGE OF ${ }^{\theta}$ THE SIMULATION DIAGRAM OF $D$ 


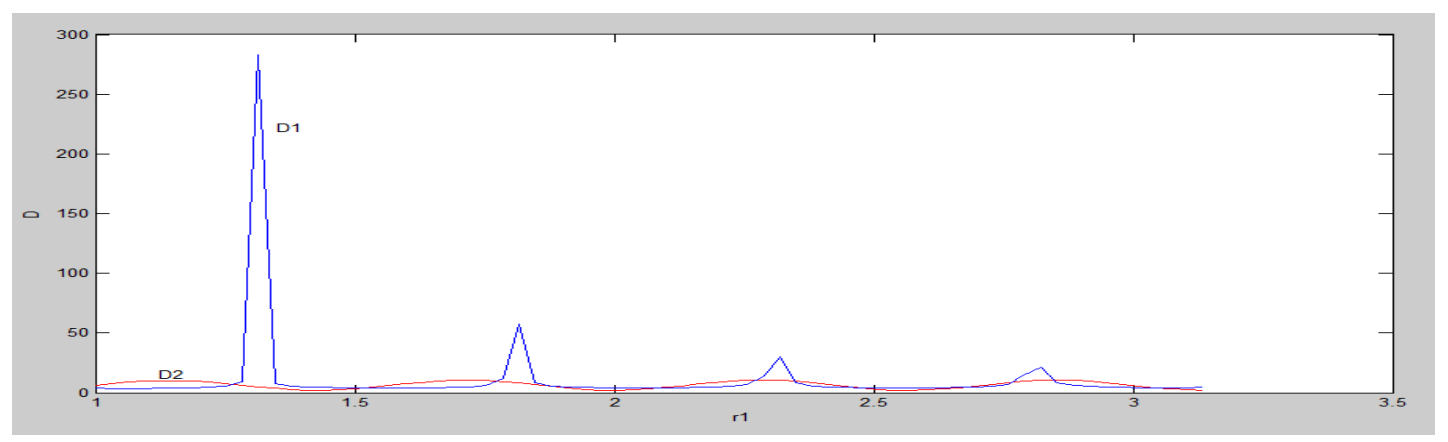

FIGURE III. WITH THE CHANGE OF ${ }^{{ }^{1}}$ THE SIMULATION DIAGRAM OF $D$

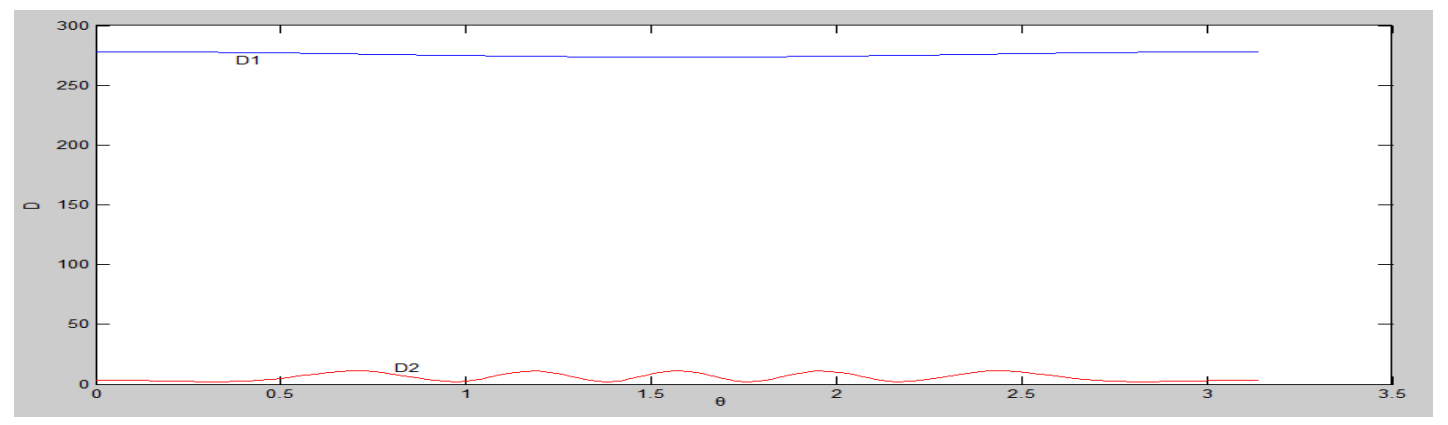

FIGURE IV. ${ }^{r_{1}=1.3142}$ WITH THE CHANGE OF ${ }^{\theta}$ THE SIMULATION DIAGRAM OF $D$

When the ${ }^{\theta_{0}, \varphi_{0}, \theta, r_{0}}$ take fixed value using Matlab software to draw the simulation diagram of ${ }^{D_{1}, D_{2}}$ with the change of $\theta$ is shown in figure III. Through figure III known when ${ }^{r_{1}}$ get 1.2681 1.5137.1.7737 2.0867、

value in $2.2740 \sim 2.3493 .2 .7663 \sim 2.8481$ these areas directivity coefficient $D_{1}$ far bigger than $D_{2}$ and when ${ }^{r_{1}}$ get value $1.3142 D_{1}$ the maximum value 283.11 is 51.47 times of ${ }^{D_{2}}=5.5$. When $r_{1}$ get value $1.8168 D_{1=57.164}$ is six times of $D_{2}=9.5$. When $r_{1}$ get value $2.3195^{D_{1}}$ is 2.71 times of ${ }^{D_{2}}=9.5$.

When the $\theta_{0}=0, \varphi_{0}=0, r_{0}=0, r_{1}=1.3142$ take fixed value using Matlab software to draw the simulation diagram of ${ }^{D_{1}, D_{2}}$ with the change of $\theta$ is shown in figure IV. According to the figure IV know when get $\theta_{0}=0, \varphi_{0}=0, r_{0}=0, r_{1}=1.3142$, wherever the direction function of the angular momentum of the binary array antenna directivity $D_{1}$ with ${ }^{\theta}$ varying from 0 to ${ }^{\pi}$ far

bigger than $D_{2}$ of the figure function of not angular momentum of binary antenna array directivity.

\section{CONCLUSION}

In this paper, using the eq.(2) deduce the direction function of angular momentum the directivity coefficients of binary array antenna, and using the Matlab software simulation to compare $D_{1}$ and $D_{2}$ get the following conclusion.

1) When the $\theta_{0}, \varphi_{0}, r_{0}, r_{1}$ set value, in certain area the direction function of angular momentum the directivity coefficients of the binary array antenna ${ }^{D_{1}}$ with ${ }^{\theta}$ varying from 0 to $\pi$ bigger than $D_{2}$ the direction function of not angular momentum the directivity of coefficients binary antenna array.

2) when the $\theta_{0}, \varphi_{0}, \theta, r_{0}$ set value, in certain area $D_{1}$ with ${ }^{{ }^{1}}$ varying from 0 to $\pi$ bigger than $D_{2}$.

3) when the $\theta_{0}=0, \varphi_{0}=0, r_{0}=0, r_{1}=1.3142$ set value, wherever $D_{1}$ with ${ }^{\theta}$ varying from 0 to $\pi$ far bigger than $D_{2}$.

Conclusion 3) has important meaning for actual antenna design, as long as taking $r_{1}=1.3142$ the directivity coefficients of binary antenna array ${ }^{D_{1}}$ can bigger dozens of times than $D_{2}$ conventional the directivity coefficients of binary antenna array.

\section{REFERENCES}

[1] X. B. Hou, "Space solar power stations," unpublished.

[2] Tseng C Y, Griffiths L J.A simple algorithm to achieve desired patterns for arbitrary arrays [J].IEEE Trans Sig- nal Processing, 1992,40(11):2737 - 2746.

[3] Olen C A, Compton R T.A numerical pattern synthesis algorithm for arrays[ J].IEEE Trans Antennas Propagat, 1990, 38(10):1666 -1676.

[4] Y C Sheng, R H Jin. A speedy array pattern synthesis method based on pattern expansion and FFT [J] . Ch-inese Journal of Radio Science, 2003, 18 ( 5) : 540-544

[5] Wang F, Yang R, Frank C.A new algorithm for array patern synthesis using the recursive. Squares method [J].IEEE Signal Processing. Lett,2003,10 (8):235-238.

[6] Y Fan, R H Jin. Pattern synthesis of antennas based on a novel genetic algorithm. [J]. Chinese Journal of Radio Science, 2004, 19(2): 182-186. 
[7] Sanzgiri S M, Bulter J K. Constrained optimization of the performance indices of arbitrary array antennas J.IEEE Trans. Antennas Propag., 1971, 19(4): 493-498.

[8] Y F Chen, X Z Huang. "Super directional antennao ptimization synthesis method research,”J. In the national conference proceeding so fmilli meter microwave.2007, c:20-27.

[9] J. Y. Zen, Quantum mechanics tutorial,2rd ed., Beijing: science press, 2008,pp.250- 251

[10] Z.H. Xue,W. M. Li,and W. Ren, Antenna array analysis and synthesis,1rd ed., Beijing: Beijing university of aeronautics and astronautics press, 2011,pp.1-20. 